\title{
Total Body PET: Exploring New Horizons
}

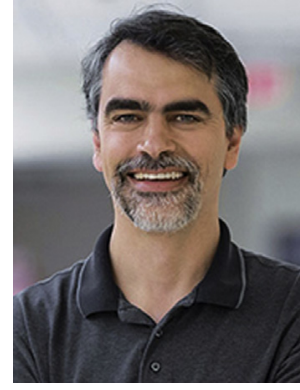

Ramsey D. Badawi, PhD

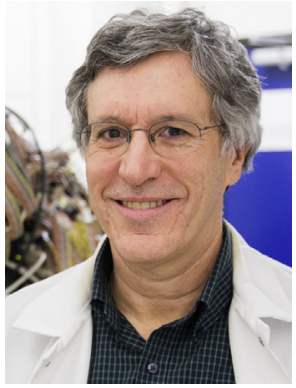

Joel S. Karp, PhD

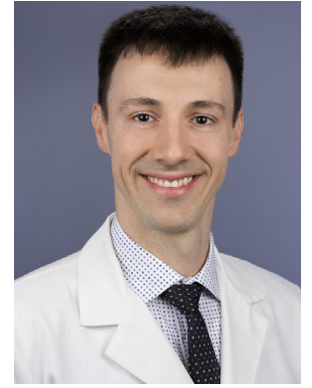

Lorenzo Nardo, MD, PhD

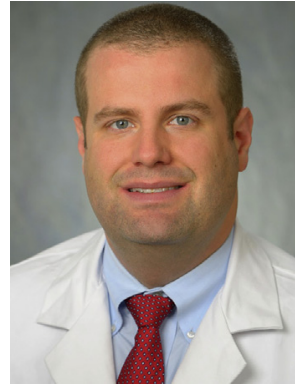

Austin R. Pantel, MD, MSTR Editors

In this special issue of PET Clinics, we provide the readers with a complete update of where total body (TB) PET stands at the present time, together with some views on future applications for this transformative technology. TB PET is a 30 -yearold idea finally brought to fruition in 2018 , by the EXPLORER Consortium, with the beginning of human imaging with TB PET/CT scanners in the United States and in China. The EXPLORER Consortium has developed 2 different scanner designs with axial fields-of-view (AFOV) significantly beyond that of existing systems: the UEXPLORER, manufactured by United Imaging Healthcare, and the PennPET Explorer, manufactured at the University of Pennsylvania. At the time of this writing, TB PET has been in clinical operation for a little over a year at the University of California at Davis, at Zhongshan Hospital, and at several other sites in China with the UEXPLORER scanner, and for research investigations at the University of Pennsylvania with the PennPET Explorer scanner. The clinical and research potentials are just beginning to be explored.

TB PET is characterized by exceptional signal collection efficiency resulting from close-tomaximal geometric coverage, and by the ability to simultaneously collect signal from multiple organs and potentially the entire body. These features allow the clinician, or research investigator, to select a variety of protocols not available before, including ultrafast or ultralow dose scanning, scanning with unprecedented image quality, scanning at much later time points after radiotracer injection, and, for the first time with any imaging modality, the ability to simultaneously capture radiotracer kinetics across the entire body. All these capabilities have now been demonstrated in the early use of these instruments, and examples of such studies are presented in this issue. Many of the potential applications of TB PET imaging are discussed in the following articles, including oncology and metabolic disease, atherosclerosis, musculoskeletal disease, infection, cardiac disease, and neurologic disease. Perhaps even more transformative, these capabilities may enable wider application of PET scanning in the management and understanding of human health; some of these possibilities are discussed herein.

We expect that the enthusiasm for TB PET imaging will lead to the introduction of other systems that offer similar, or possibly superior performance compared with the 2 EXPLORER designs. In particular, it is already becoming clear that we will see a range of AFOV for new instruments since it is not yet known what the optimal axial coverage should be for a TB PET instrument, or if indeed there is a single such optimum. Ultimately, the primary use of the scanner should dictate its configuration, which may also be constrained by cost. The many applications discussed in the following 
articles provide guidance and inspiration for future designs.

TB PET is in its infancy and yet has already shown quite astonishing results. This issue of PET Clinics sets the stage for the next phase of exploration.

Ramsey D. Badawi, PhD Department of Radiology University of California Davis 4860 Y Street, Suite 3100 Sacramento, CA 95817, USA Joel S. Karp, PhD Department of Radiology University of Pennsylvania 3620 Hamilton Walk John Morgan Building - Room \#154 Philadelphia, PA 19104-6112, USA
Lorenzo Nardo, MD, PhD Department of Radiology University of California Davis 4860 Y Street, Suite 3100 Sacramento, CA 95817, USA

Austin R. Pantel, MD, MSTR Department of Radiology University of Pennsylvania 3400 Spruce Street 1 Silverstein, Suite 130 Philadelphia, PA 19104, USA

E-mail addresses: rdbadawi@ucdavis.edu (R.D. Badawi) joelkarp@pennmedicine.upenn.edu (J.S. Karp) Inardo@ucdavis.edu (L. Nardo) austin.pante|@pennmedicine.upenn.edu

(A.R. Pantel) 